\title{
Probing the BCS-BEC crossover with persistent currents
}

\author{
Giovanni Pecci $\odot,{ }^{1}$ Piero Naldesi, ${ }^{1}$ Luigi Amico, ${ }^{2,3,4,5}$ and Anna Minguzzi ${ }^{1}$ \\ ${ }^{1}$ Université Grenoble Alpes, CNRS, LPMMC, 38000 Grenoble, France \\ ${ }^{2}$ Quantum Research Centre, Technology Innovation Institute, P.O. Box 9639, Abu Dhabi, United Arab Emirates \\ ${ }^{3}$ CNR-IMM and INFN-Sezione di Catania, Via S. Sofia 64, 95127 Catania, Italy \\ ${ }^{4}$ Centre for Quantum Technologies, National University of Singapore, 3 Science Drive 2, Singapore 117543, Singapore \\ ${ }^{5}$ LANEF Chaire d'Excellence, Université Grenoble-Alpes and CNRS, F-38000 Grenoble, France
}

(Received 13 October 2020; accepted 9 July 2021; published 14 September 2021)

\begin{abstract}
We study the persistent currents of an attractive Fermi gas confined in a tightly confining ring trap and subjected to an artificial gauge field all through the BCS-BEC crossover. At weak attractions, on the BardeenCooper-Schrieffer (BCS) side, fermions display a parity effect in the persistent currents, i.e., their response to the gauge field is paramagnetic or diamagnetic depending on the number of pairs on the ring. At resonance and on the Bose-Einstein condensate (BEC) side of the crossover we find a doubling of the periodicity of the ground-state energy as a function of the artificial gauge field and disappearance of the parity effect, indicating that persistent currents can be used to infer the formation of tightly bound bosonic pairs. Our predictions can be accessed in ultracold atom experiments through noise interferograms.
\end{abstract}

DOI: 10.1103/PhysRevResearch.3.L032064

Introduction. A gas of weakly attractive spin- $\frac{1}{2}$ fermions can form bound pairs with opposite spin and condense into the Bardeen-Cooper-Schrieffer (BCS paired regime. On the other hand, particles with integer spin can display Bose-Einstein condensation (BEC). Despite BCS pairing and BEC are two distinct physical phenomena, they have been intensively studied as two different regimes that may occur in the same system. In the BCS regime, the correlation length of the bound pairs is large compared with the typical interparticle distance; in the BEC regime, instead, the pairs are tightly bound in the real space and the pair correlation length is much smaller than the distance between the particles. The evolution between the two regimes is called BCS-BEC crossover [1]. It plays an important role in different contexts, ranging from nuclear [2,3] to condensed matter physics [4,5]. With the advent of ultracold atoms quantum technology, the BCS-BEC crossover has been studied in the laboratory with unprecedented control of the physical conditions, making it possible to test important aspects of the theories developed so far [6-8].

Recently, important progress has been achieved in the field, allowing coherent manipulation of atoms in trapping potentials with wide ranges of intensities and shapes, in an unprecedented precise manner [9-13]. Atomtronics exploits such remarkable progress to realize matter-wave circuits of ultracold atoms manipulated in magnetic or laser-generated guides [14-16]. In particular, harnessing current states in an explicit way, atomtronics effectively widens the scope of

Published by the American Physical Society under the terms of the Creative Commons Attribution 4.0 International license. Further distribution of this work must maintain attribution to the author(s) and the published article's title, journal citation, and DOI. quantum simulators and emulators to probe quantum phases of matter. Specifically, in the spirit of solid-state physics I$V$ (current-voltage) characteristics, the different many-body quantum regimes are characterized in terms of the current flowing through the cold atomic system. Here, we take the latter view to study the BCS-BEC crossover: we show how the persistent current in attracting fermionic systems confined in ring-shape lattice potential and pierced by an artificial gauge field provides a way to tell apart the BCS and BEC regimes. Modeling the $\mathrm{BCS}-\mathrm{BEC}$ crossover in $1 \mathrm{D}$ needs to be done differently than in the $3 \mathrm{D}$ case $[17,18]$. We follow the same strategy as in [19]: we describe the system on the BCS side using a model of fermions with attractive contact interactions. At resonance, it corresponds to a Tonks-Girardeau gas of hard-core bosonic pairs [20]. The BEC side of the crossover is described by a bosonic model for the pairs with contact interactions, to which the attractive fermionic model is continuously connected. By applying exact Bethe ansatz methods corroborated by density matrix renormalization group (DMRG) simulations, we gain access to all regimes of interactions ranging from weak to strong attractions.

Our main results are summarized in Figs. 1 and 2. We rely on a theorem due to Leggett [21] to demonstrate that the BCS to $\mathrm{BEC}$ crossover is marked by clear features of the periodicity of the persistent currents. Accordingly, assuming that the total number of particles is $N=2 n$, the persistent current of a gas of interacting spin- $\frac{1}{2}$ fermions is predicted to be parity dependent: for even number $n$ of pairs of fermions, the system behaves as a paramagnet with a nonvanishing persistent current at zero effective magnetic field; for odd $n$ instead, the system behaves as a diamagnet (vanishing persistent current at zero field). For our specific problem, we find that while the persistent current displays clear parity dependence in the BCS 
(a)
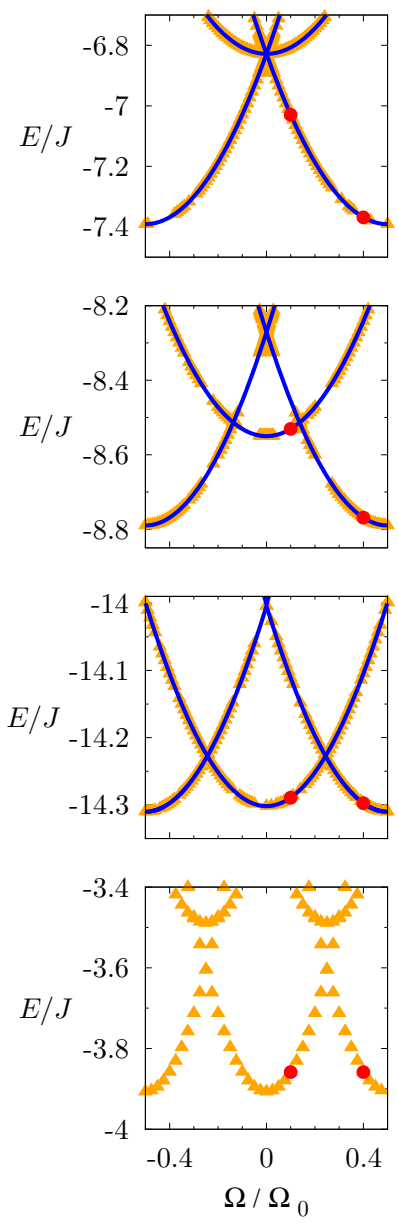

(b)
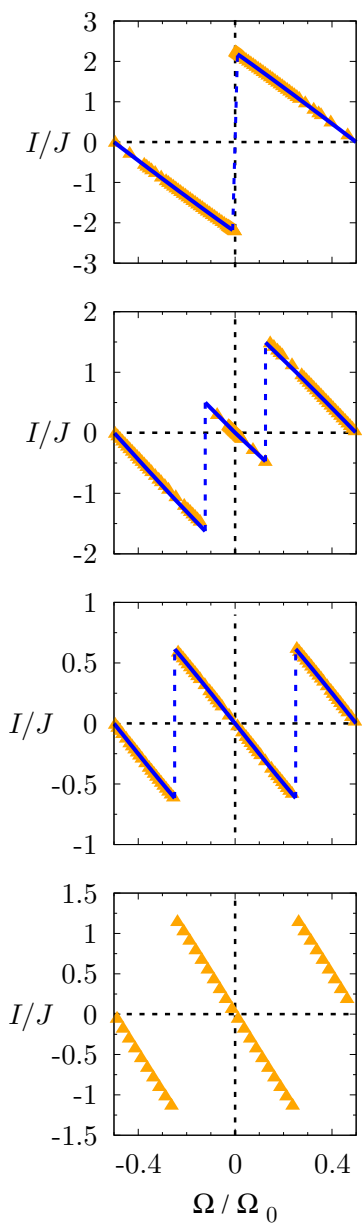

(c)
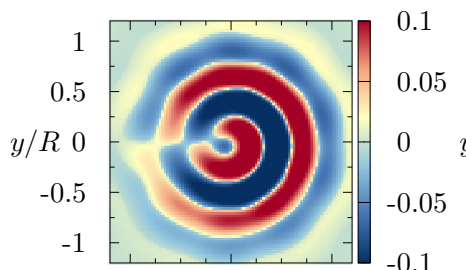

$-0.1$
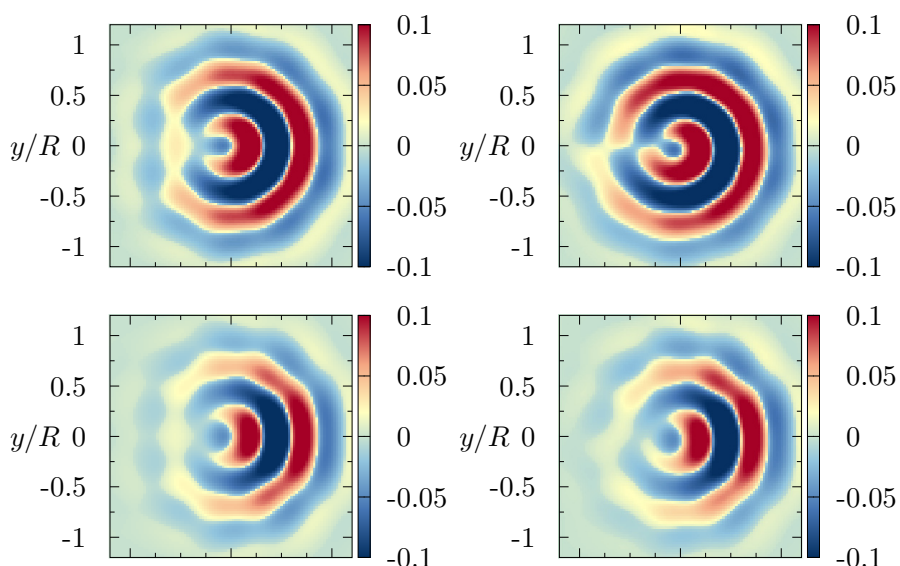

0.1
0.05
0
-0.05
-0.1
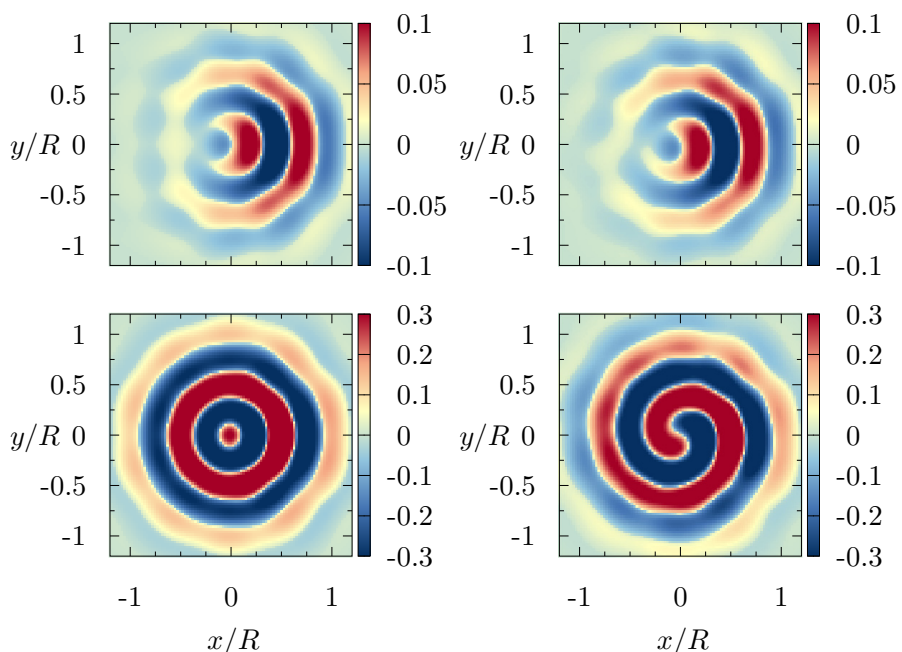

FIG. 1. Column (a): energies vs $\Omega$ for $N=4, N_{s}=8$ from Bethe ansatz (blue line) and DMRG (yellow triangles). In each column, the first three panels from the top correspond to $U / J=0,-2,-6$, respectively. The last line describes the BEC regime for $U_{B} / J_{B}=1-$ here only DMRG data are available since the model is not integrable. Column (b): persistent current as a function of the flux for the same set of parameters, obtained as the derivative of the ground-state energy in column (a). Columns (c) and (d): noise correlator for $N=4$ and $N_{s}=10$ for $\Omega / \Omega_{0}=0.1$ and $\Omega / \Omega_{0}=0.4$, respectively, indicated by red circles in column (a). The correlators are all evaluated in $r^{\prime}=(R, 0)$ and $t=0.3 \omega_{0}^{-1}$, where $\omega_{0}$ is the frequency of each lattice well. The noise correlator is expressed in units of $1 / R^{2}$. A circulating state is characterized by a spiral-like correlator, not symmetric by inversion with respect to the $y=0$ axis.

regime, the latter is washed out for strongly attracting pairs, indicating that at resonance and in the BEC regime, fermionic pairs behave as pointlike bosons, which are predicted not to show parity effects [22,23]. Finally, inspired by a procedure developed for bosonic condensates [24-27], we propose a protocol to evidence the parity effect in the persistent current by noise correlations, based on the self-heterodyne detection of the phase of the many-body wave function.

The model. We consider a gas of degenerate fermions confined in a lattice ring of radius $R$ and pierced by an effective gauge field. Such system is a paradigmatic example of an atomtronic circuit $[15,16]$. The artificial magnetic field can be applied in several ways; for example, by stirring the condensate, by phase imprinting, or two-photon Raman transitions [29]. To address the whole BCS-BEC crossover, we exploit the possibility to tune the interaction strength in ultracold atoms, e.g., across a confinement-induced resonance [30,31]. To describe the BCS side of the crossover, we use the one-dimensional attractive Fermi-Hubbard Hamiltonian, which, for an even number $N$ of particles on a lattice of $N_{s}$ sites, reads

$$
\begin{aligned}
\hat{\mathcal{H}}_{F H}= & -J \sum_{j=1}^{N_{s}} \sum_{\sigma=\uparrow, \downarrow}\left(e^{i(2 \pi / N s)\left(\Omega / \Omega_{0}\right)} c_{j, \sigma}^{\dagger} c_{j+1, \sigma}+\text { H.c. }\right) \\
& +U \sum_{j=1}^{N_{s}} n_{j, \uparrow} n_{j, \downarrow}
\end{aligned}
$$

where $U<0, J$ is the tunnel amplitude, $\Omega$ is the rotation frequency induced by the artificial gauge field, and $\Omega_{0}=\hbar / m R^{2}$. The Hamiltonian (1) is solvable by Bethe ansatz [32,33] with many-body eigenvalues in the form $E=-2 J \sum_{j} \cos k_{j}$. The quantities $k_{j}$ are the so-called charge rapidities: we set them as $k_{j, \pm}=p_{j} \pm i v_{j}$. In thermodynamic limit they correspond to two-dimensional $k$ strings in which all the charge rapidities are in the form $\sin \left(k_{j, \pm}\right)=\Lambda_{j} \pm i U / 4 J$. A nonvanishing 
(a)
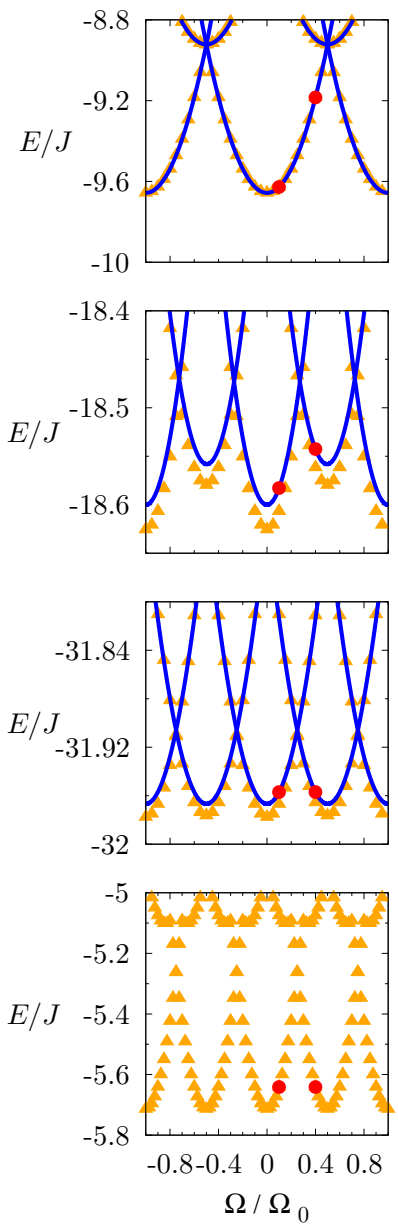

(b)
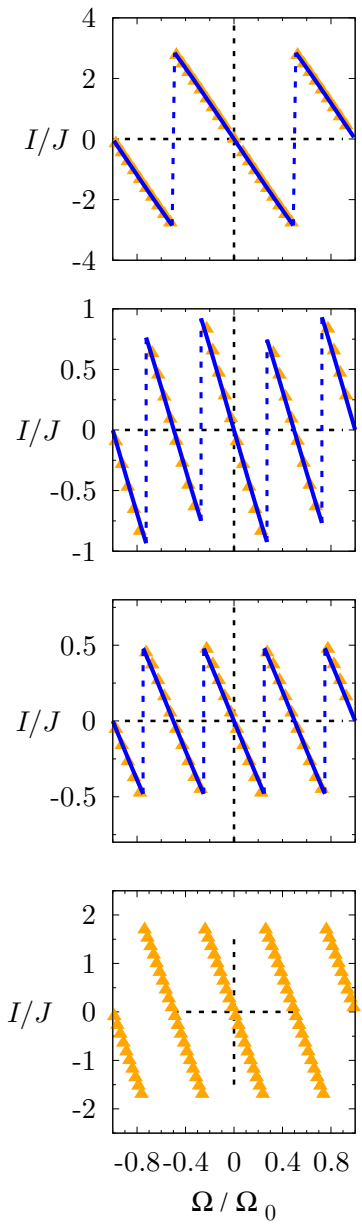

(c)
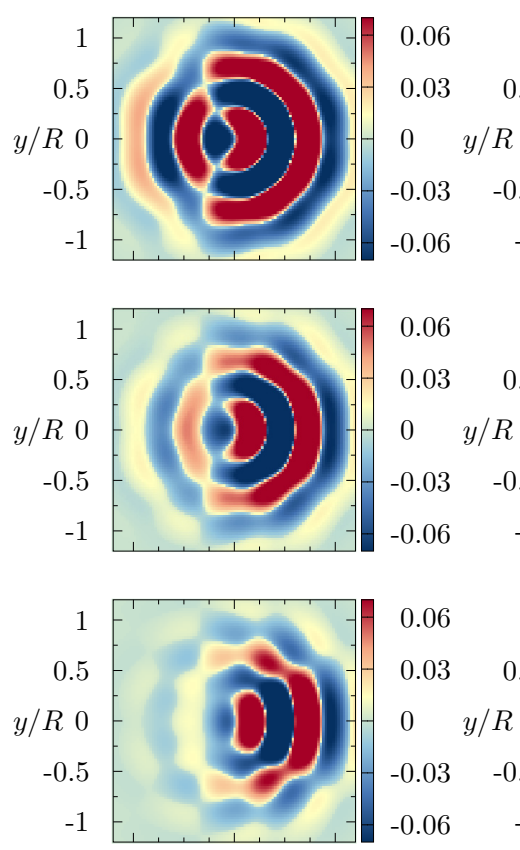

$\begin{array}{lr}0.06 & \\ 0.03 & 0.5 \\ 0 & y / R \\ -0.03 & -0.5 \\ -0.06 & -1\end{array}$
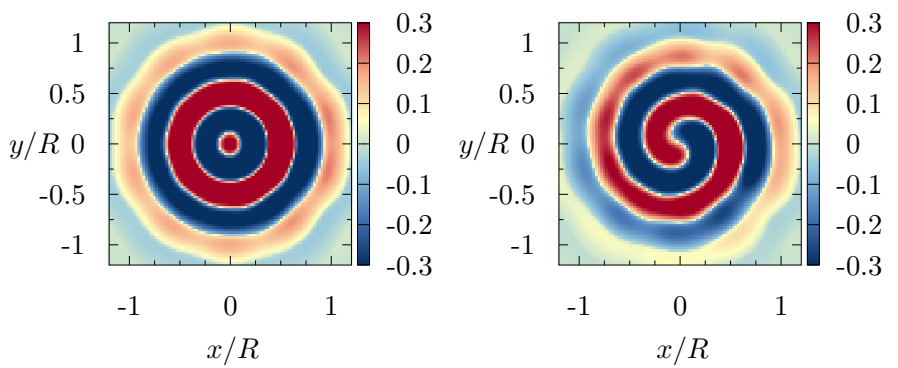

FIG. 2. Energies, persistent current, and noise correlators as in Fig. 1 here studied for $N=6$, and for $U / J=0,-5,-10$ (from top to bottom) [28]. The last line refers to the bosonic case for $U_{B} / J_{B}=1$. All the other parameters are the same as in Fig. 1.

imaginary part of the rapidities indicates the formation of bound states. Excitations on top of the ground states are obtained either by pair breaking (gapped spin excitations), by pair rearrangements (gapless charge excitations), or by combinations thereof [34]. We note that solutions deviating from the $k$-string form occur if $U N_{s} / J$ is not sufficiently large. This may happen on a lattice ring at very weak interactions. We provide a solution to this model at any interaction strength and in the total spin zero sector.

In the small- $|U|$ limit, the connection between the onedimensional (1D) Hubbard model and BCS theory is well established [35,36].

The BCS regime emerges as the limit where the binding energy is smaller than the kinetic energy of the particles, and the bound states are localized on spatial scales much larger than the average interparticle distances [37]. The known BCS exponential dependence of the energy gap on interaction was also obtained [38].

For strongly attractive interactions, the Bethe ansatz solution indicates the formation of a Tonks-Girardeau gas of hard-core bosons. Hence, in the spirit of [19], we describe the BEC side of the crossover by a bosonic Hubbard model, where bosons correspond to the fermionic pairs,

$$
\begin{aligned}
\hat{\mathcal{H}}_{B H}= & -J_{B} \sum_{j=1}^{N_{s}}\left(e^{i(2 \pi / N s)\left(\Omega / \Omega_{0}\right)} b_{j}^{\dagger} b_{j+1}+\text { H.c. }\right) \\
& +\frac{U_{B}}{2} \sum_{j=1}^{N_{s}} n_{j}\left(n_{j}-1\right) .
\end{aligned}
$$

Here $J_{B}$ is the tunnel amplitude for pairs and $U_{B}$ is the pairpair interaction strength [39]. We remark that different from the Fermi-Hubbard model, the bosonic one is not integrable $[40,41]$ : there is no exact solution for this model. To address the whole BCS-BEC crossover, in our work we combine Bethe ansatz calculations and DMRG simulations to calculate ground- and excited-state energy branches as a function of $\Omega$. DMRG is also used to calculate noise correlators.

Parity effects of the persistent current. We obtain the persistent current from the ground-state energy branch according to $I=-\Omega_{0} \partial E_{G S} / \partial \Omega$. For zero and weak interactions, the persistent current is a periodic function of the flux with period $\Omega_{0}$. Its behavior markedly depends on the parity of the number of pairs $N / 2$ (see Figs. 1 and 2): for even $N / 2$, the ground-state 
energy displays a global maximum for $\Omega=0$ (paramagnetic behavior); for $o d d N / 2$, instead, the ground-state energy has a global minimum for $\Omega=0$ (diamagnetic behavior). Such effect emerges by comparing the first two columns of Figs. 1 and 2. Remarkably, the parity dependence of the persistent current disappears for strong enough interactions: upon increasing interactions the ground-state energy displays a superlattice structure as the energy of the excited states decreases, leading to the doubling of its periodicity and the suppression of the parity effects in the persistent current at resonance. On the BEC side the periodicity of the ground-state energy is $\Omega_{0} / 2$, corresponding to the quantum of flux of a pair, for any $U_{B}$. We find that the doubling of periodicity of the current is a clear signature of the formation of pairs. This was originally suggested by Byers and Yang [42] for BCS superconducting rings. Our results elucidate their predictions by an exact calculation and extend it to the whole BCS-BEC crossover.

The decrease of the energy of the excited states is also at the origin of the change of sign in the curvature of the ground-state energy at zero flux observed in the disordered Fermi-Hubbard model for even $N / 2$ [43]: disorder smooths the cusps at weak interactions yielding a negative curvature, while a positive curvature is found at strong attractions as in the clean case.

Readout: Interferograms and noise correlations. We next suggest a protocol to probe the BCS-BEC crossover in cold atoms setup. In analogy to the approach carried out for bosonic systems, we propose an interferometric detection of the current by studying the interference pattern arising from the coexpansion of the gas on the ring and a degenerate gas placed at the center $C$ of the ring [39]. In our protocol we assume the free expansion of the gas. Interaction effects arising from inelastic collisions are expected to be negligible in our low density regime [44]. For higher densities, specific fast ramping protocol leading the system to the BEC side could be adopted to improve the visibility of the interferograms $[8,45,46]$. We model the gas in the center as a single site, and assume that no hopping between the ring and the central site can occur. We then study density-density correlations at equal time, which on the BCS side reads $\sum_{\rho, \sigma=\uparrow, \downarrow}\left\langle n_{\rho}(r, t) n_{\sigma}\left(r^{\prime}, t\right)\right\rangle$, where $n_{\sigma}(r, t)=\Psi_{\sigma}^{\dagger}(r, t) \Psi_{\sigma}(r, t)$ is the density operator for the spin component $\sigma, \Psi_{\sigma}(r, t)$ being the fermionic field operator. On the BEC side the first nontrivial correlator is the one associated to density of pairs $n_{B}$ [47], i.e., $\left\langle n_{B}(r, t) n_{B}\left(r^{\prime}, t\right)\right\rangle$.

In order to enhance the visibility of the correlator, arising from the low density of the system, we generalize the method devised in [47]: we find that the only terms producing a nontrivial interference pattern are the ones describing the correlations between the expanding ring and center:

$$
\begin{aligned}
\tilde{G}\left(r, r^{\prime}, t\right)= & -\tilde{G}_{0}\left(r, r^{\prime}, t\right)+\sum_{\rho, \sigma=\uparrow, \downarrow}\left[\left\langle n_{\rho}(r, t) n_{\sigma}\left(r^{\prime}, t\right)\right\rangle\right. \\
& -\left\langle n_{\rho}(r, t)\right\rangle_{\text {ring }}\left\langle n_{\sigma}\left(r^{\prime}, t\right)\right\rangle_{\mathrm{C}} \\
& -\left\langle n_{\rho}(r, t)\right\rangle_{\mathrm{C}}\left\langle n_{\sigma}\left(r^{\prime}, t\right)\right\rangle_{\text {ring }} \\
& \left.-\left\langle n_{\rho}(r, t) n_{\sigma}\left(r^{\prime}, t\right)\right\rangle_{\text {ring }}-\left\langle n_{\rho}(r, t) n_{\sigma}\left(r^{\prime}, t\right)\right\rangle_{\mathrm{C}}\right],
\end{aligned}
$$

where $\tilde{G}_{0}\left(r, r^{\prime}, t\right)=w_{\mathrm{C}}^{*}(r, t) w_{\mathrm{C}}\left(r^{\prime}, t\right) \sum_{j=1}^{N_{s}} w_{j}(r, t) w_{j}^{*}\left(r^{\prime}, t\right)$ has also been subtracted, with $w_{j}(r, t)$ the expanding Wannier function on the site $j$ at time $t$ [39], and we have set $\Psi_{\sigma}(r)=w_{\mathrm{C}}(r) c_{\mathrm{C}, \sigma}+\sum_{j=1}^{N_{s}} w_{j}(r) c_{j, \sigma}$. The $\tilde{G}_{0}$ term corresponds to the one-body density matrix of a noninteracting Fermi gas for a completely filled lattice, i.e., when it forms a band insulator. On the BEC side, a definition analogous to Eq. (3) involving the bosonic density is used. We remark that the above approximation leads to non-Hermitian $\tilde{G}\left(r, r^{\prime}, t\right)$ [39], hence in the following, we focus only on the square root of the real part of $\tilde{G}\left(r, r^{\prime}, t\right)$, i.e., $G\left(r, r^{\prime}, t\right)=$ $\operatorname{sgn}\left(\operatorname{Re}\left[\tilde{G}\left(r, r^{\prime}, t\right)\right]\right) \sqrt{\operatorname{Re}\left[\tilde{G}\left(r, r^{\prime}, t\right)\right]}$.

We study the interference pattern for systems with even and odd number of pairs in columns (c) and (d) of Figs. 1 and 2. In the small $\Omega$ case, at weak interactions we see a spiral image with a dislocation indicating nonzero current for even $N / 2$, while there is no current for odd $N / 2$ as the figure is symmetric by reflection along the $y=0$ axis. The shape of the interferograms is due to the different contributions of the single-particle orbitals constituting the Fermi sphere [48]. In contrast, at strong interactions, the images for $N / 2$ even or odd are both symmetric with respect to the $y=0$ axis, indicating that the current vanishes regardless of the parity of the number of pairs. We also study the correlation in the interference pattern for $\Omega / \Omega_{0}$ slightly below the degeneracy point $\Omega / \Omega_{0}=1 / 2$. Comparing columns (c) and (d) of both Figs. 1 and 2 , we see that at zero interactions the current is the same in the two cases, consistently with the fact that the two values of flux are on the same period of the ground-state energy. At strong interactions, close to resonance, the images are more blurred by the reduced phase coherence. Nevertheless, we see that the correlation functions of column (d) display nonmirror symmetric, spiral-like features, indicating the presence of a current state and the doubling of the periodicity. Finally, on the BEC side, the coherence producing well defined spirals arises from the quasicondensation of pairs.

Conclusions and outlook. We studied the persistent current of a Fermi gas confined in a mesoscopic ring-shaped lattice subjected to an artificial magnetic field all through the BCS-BEC crossover. We described the system through attractive Fermi and repulsive Bose-Hubbard models, using both the exact solution by Bethe ansatz and DMRG. We demonstrated that the persistent current displays distinctive features in the various interaction regimes. At weak interactions (BCS regime), the persistent current is a periodic function of the single-particle flux quantum, displaying some modulations due to the superlattice structure of the groundstate energy. Such phenomenon indicates the onset of pairing correlations building up between up and down spins. The BCS regime is characterized by parity-dependent persistent currents: while for odd number of pairs the system has a diamagnetic response, for even number of pairs the system has a paramagnetic response. Remarkably, the parity effect is washed out at resonance and in the BEC regime. In these regimes, the persistent current is periodic with a two-particle flux quantum, providing a clear signature of the formation of bound pairs. To experimentally monitor the features of the persistent current described above, we let the gas coexpand with a reference gas placed in the center and we study the 
noise correlations in the interference pattern. Quite remarkably, such interferometric analysis works also in the BCS regime, where the phase coherence ensured by the fermionic pairs is lower than in the BEC one.

Our work provides clear evidence that the response of a mesoscopic size system to an artificial gauge field is a relevant tool to study the BCS-BEC crossover. Our approach is fully general and readily applicable to other models, e.g., the bosonfermion one [49], as well as to other systems, such as high $T_{c}$ superconductors [50,51].

Acknowledgments. We thank T. Haug and C. Salomon for discussions. The Grenoble LANEF framework (ANR10-LABX-51-01) is acknowledged for its support with mutualized infrastructure.
[1] G. C. Strinati, P. Pieri, G. Röpke, P. Schuck, and M. Urban, The BCS-BEC crossover: From ultra-cold Fermi gases to nuclear systems, Phys. Rep. 738, 1 (2018).

[2] M. Baldo, U. Lombardo, and P. Schuck, Deuteron formation in expanding nuclear matter from a strong coupling BCS approach, Phys. Rev. C 52, 975 (1995).

[3] R. F. Bishop, K. A. Gernoth, N. R. Walet, and Y. Xian, Recent Progress in Many-Body Theories (World Scientific, Singapore, 2000), Vol. 3.

[4] M. Randeria, J.-M. Duan, and L.-Y. Shieh, Bound States, Cooper Pairing, and Bose Condensation in Two Dimensions, Phys. Rev. Lett. 62, 981 (1989).

[5] R. Micnas, J. Ranninger, and S. Robaszkiewicz, Superconductivity in narrow-band systems with local nonretarded attractive interactions, Rev. Mod. Phys. 62, 113 (1990).

[6] M. Randeria, W. Zwerger, and M. Zwierlein, in The BCS-BEC Crossover and the Unitary Fermi Gas (Springer, New York, 2012), pp. 1-32.

[7] M. Inguscio, W. Ketterle, and C. Salomon, Ultra-Cold Fermi Gases (IOS Press, Clifton, VA, 2008), Vol. 164.

[8] G. Valtolina, A. Burchianti, A. Amico, E. Neri, K. Xhani, J. A. Seman, A. Trombettoni, A. Smerzi, M. Zaccanti, M. Inguscio et al., Josephson effect in fermionic superfluids across the BECBCS crossover, Science 350, 1505 (2015).

[9] W. Kwon, G. Del Pace, R. Panza, M. Inguscio, W. Zwerger, M. Zaccanti, F. Scazza, and G. Roati, Strongly correlated superfluid order parameters from dc Josephson supercurrents, Science 369, 84 (2020).

[10] H. Rubinsztein-Dunlop, A. Forbes, M. V. Berry, M. R. Dennis, D. L. Andrews, M. Mansuripur, C. Denz, C. Alpmann, P. Banzer, T. Bauer et al., Roadmap on structured light, J. Opt. 19, 013001 (2016).

[11] J.-P. Brantut, J. Meineke, D. Stadler, S. Krinner, and T. Esslinger, Conduction of ultracold fermions through a mesoscopic channel, Science 337, 1069 (2012).

[12] F. Serwane, G. Zürn, T. Lompe, T. Ottenstein, A. Wenz, and S. Jochim, Deterministic preparation of a tunable few-fermion system, Science 332, 336 (2011).

[13] G. Zürn, A. N. Wenz, S. Murmann, A. Bergschneider, T. Lompe, and S. Jochim, Pairing in Few-Fermion Systems with Attractive Interactions, Phys. Rev. Lett. 111, 175302 (2013).

[14] L. Amico, M. Boshier, G. Birkl, A. Minguzzi, C. Miniatura, L. C. Kwek, D. Aghamalyan, V. Ahufinger, N. Andrei, A. S. Arnold et al., Roadmap on atomtronics, arXiv:2008.04439.

[15] L. Amico, G. Birkl, M. Boshier, and L.-C. Kwek, Focus on atomtronics-enabled quantum technologies, New J. Phys. 19, 020201 (2017).
[16] R. Dumke, Z. Lu, J. Close, N. Robins, A. Weis, M. Mukherjee, G. Birkl, C. Hufnagel, L. Amico, M. G. Boshier et al., Roadmap on quantum optical systems, J. Opt. 18, 093001 (2016).

[17] E. Burovski, N. Prokof'ev, B. Svistunov, and M. Troyer, Critical Temperature and Thermodynamics of Attractive Fermions at Unitarity, Phys. Rev. Lett. 96, 160402 (2006).

[18] C.-C. Chien, Y. He, Q. Chen, and K. Levin, Superfluid-insulator transitions at noninteger filling in optical lattices of fermionic atoms, Phys. Rev. A 77, 011601(R) (2008).

[19] J. N. Fuchs, A. Recati, and W. Zwerger, Exactly Solvable Model of the BCS-BEC Crossover, Phys. Rev. Lett. 93, 090408 (2004).

[20] T. Iida and M. Wadati, Exact analysis of $\delta$-function attractive fermions and repulsive bosons in one-dimension, J. Phys. Soc. Jpn. 74, 1724 (2005).

[21] A. J. Leggett, in Granular Nanoelectronics, edited by D. K.Ferry, J. R. Barker, and C. Jacoboni (Springer, US, Boston, 1991), pp. 297-311.

[22] C. Schilling and R. Schilling, Number-parity effect for confined fermions in one dimension, Phys. Rev. A 93, 021601(R) (2016).

[23] M. Manninen, S. Viefers, and S. Reimann, Quantum rings for beginners II: Bosons versus fermions, Physica E 46, 119 (2012).

[24] L. Corman, L. Chomaz, T. Bienaimé, R. Desbuquois, C. Weitenberg, S. Nascimbene, J. Dalibard, and J. Beugnon, Quench-Induced Supercurrents in an Annular Bose Gas, Phys. Rev. Lett. 113, 135302 (2014).

[25] K. C. Wright, R. B. Blakestad, C. J. Lobb, W. D. Phillips, and G. K. Campbell, Driving Phase Slips in a Superfluid Atom Circuit with a Rotating Weak Link, Phys. Rev. Lett. 110, 025302 (2013).

[26] S. Eckel, F. Jendrzejewski, A. Kumar, C. J. Lobb, and G. K. Campbell, Interferometric Measurement of the Current-Phase Rrelationship of a Superfluid Weak Link, Phys. Rev. X 4, 031052 (2014).

[27] R. Mathew, A. Kumar, S. Eckel, F. Jendrzejewski, G. K Campbell, M. Edwards, and E. Tiesinga, Self-heterodyne detection of the in situ phase of an atomic superconducting quantum interference device, Phys. Rev. A 92, 033602 (2015).

[28] The slight discrepancy between DMRG and Bethe ansatz results for $U / J=-5$ is due to the string hypothesis made in the latter.

[29] J. Dalibard, F. Gerbier, G. Juzeliūnas, and P. Öhberg, Colloquium: Artificial gauge potentials for neutral atoms, Rev. Mod. Phys. 83, 1523 (2011).

[30] M. Olshanii, Atomic Scattering in the Presence of an External Confinement and a Gas of Impenetrable Bosons, Phys. Rev. Lett. 81, 938 (1998).

[31] M. Valiente and K. Mølmer, Quasi-one-dimensional scattering in a discrete model, Phys. Rev. A 84, 053628 (2011). 
[32] E. Lieb and F. Wu, Absence of Mott Transition in the 1D Hubbard Model, Phys. Rev. Lett 20, 1445 (1968).

[33] B. S. Shastry and B. Sutherland, Twisted Boundary Conditions and Effective Mass in Heisenberg-Ising and Hubbard Rings, Phys. Rev. Lett. 65, 243 (1990).

[34] N. Andrei, Integrable models in condensed matter physics, Low-Dimensional Quantum Field Theories for Condensed Matter Physicists (World Scientific Publishing Co., Singapore, 1995), 457 pages.

[35] F. Marsiglio, Evaluation of the BCS approximation for the attractive Hubbard model in one dimension, Phys. Rev. B 55, 575 (1997).

[36] X.-W. Guan, M. T. Batchelor, and C. Lee, Fermi gases in one dimension: From Bethe ansatz to experiments, Rev. Mod. Phys. 85, 1633 (2013).

[37] M. T. Batchelor, M. Bortz, X. W. Guan, and N. Oelkers, Exact results for the $1 \mathrm{D}$ interacting Fermi gas with arbitrary polarization, J. Phys.: Conf. Ser. 42, 5 (2006).

[38] V. Ya. Krivnov and A. A. Ovchinnikov, New method in the theory of a weakly ideal one-dimensional Fermi gas. correlation functions, JETP 49, 328 (1979).

[39] See Supplemental Material at http://link.aps.org/supplemental/ 10.1103/PhysRevResearch.3.L032064 for further details about the calculations.

[40] T. Choy and F. Haldane, Failure of Bethe-Ansatz solutions of generalisations of the Hubbard chain to arbitrary permutation symmetry, Phys. Lett. A 90, 83 (1982).

[41] T. Choy, Some exact results for a degenerate Hubbard model in one dimension, Phys. Lett. A 80, 49 (1980).
[42] N. Byers and C. N. Yang, Theoretical Considerations Concerning Quantized Magnetic Flux in Superconducting Cylinders, Phys. Rev. Lett. 7, 46 (1961).

[43] X. Waintal, G. Fleury, K. Kazymyrenko, M. Houzet, P. Schmitteckert, and D. Weinmann, Persistent Currents in One Dimension: The Counterpart of Leggett's Theorem, Phys. Rev. Lett. 101, 106804 (2008).

[44] C. Kohstall, S. Riedl, E. R. S. Guajardo, L. A. Sidorenkov, J. H. Denschlag, and R. Grimm, Observation of interference between two molecular Bose-Einstein condensates, New J. Phys. 13, 065027 (2011).

[45] M. Greiner, C. A. Regal, and D. S. Jin, Emergence of a molecular Bose-Einstein condensate from a Fermi gas, Nature (London) 426, 537 (2003).

[46] M. W. Zwierlein, J. R. Abo-Shaeer, A. Schirotzek, C. H Schunck, and W. Ketterle, Vortices and superfluidity in a strongly interacting Fermi gas, Nature (London) 435, 1047 (2005).

[47] T. Haug, J. Tan, M. Theng, R. Dumke, L.-C. Kwek, and L. Amico, Readout of the atomtronic quantum interference device, Phys. Rev. A 97, 013633 (2018).

[48] G. Pecci, P. Naldesi, A. Minguzzi, and L. Amico, Phase coherence in an interacting Fermi gas, arXiv:2105.10408.

[49] T. Ren and I. Aleiner, Bethe-ansatz analysis of near-resonant two-component systems in one dimension, Phys. Rev. A 99, 023611 (2019).

[50] Q. Chen, K. Levin, and J. Stajic, Applying BCS-BEC crossover theory to high-temperature superconductors and ultracold atomic Fermi gases, Low Temp. Phys. 32, 406 (2006).

[51] Y. Uemura, Bose-Einstein to BCS crossover picture for high- $T_{c}$ cuprates, Physica C 282, 194 (1997). 